\title{
Android Based Public Transportation System using GPS Technology
}

\author{
Mahind Rupali $\mathbf{N}^{1}$, Chautre Vinayak $\mathbf{G}^{\mathbf{2}}$ \\ Professor, Computer Science \& Engg, DACOE, Karad, India ${ }^{1}$ \\ Student, Computer Science \& Engg, DACOE, Karad, India ${ }^{2}$
}

\begin{abstract}
Now a day's everyone is using an android phone for variety of needs. Also in today's era android phones become the basic need. In most of the city's public transportation has increased due to increase in the population. In rural area and smaller town has triggered a greater need for the organized public transport system. Android based auto rickshaw booking system has been gaining more importance because they provide accurate information of route as well as the drivers and other required details at any anytime. The application has been divided in into two parts based on functionalities of the system. These modules is designed to build up an integrated system to cover various aspect of the android based auto rickshaw booking system. The android application includes a form which will be able to filled by customer according to his requirements. Later the driver will go through it and notify to the customer. The application is designed using location tracking using GPS technology.
\end{abstract}

Keywords: GPS, Android, LBS, Shortest Path Algorithm.

\section{INTRODUCTION}

Auto Rickshaw services in Karad city are predominantly unorganized in nature, wherein services are provided by individual providers and operators competing against each other for the passenger market. This structure, coupled with an improper governance framework, has created significant problems for both drivers and passengers, and is resulted in negative externalities in the economics, environmental and social realms.

The trigger behind Auto Rickshaw Services initiative was a bitter personal experience of our project members with auto rickshaw driver's overcharging and rudeness at the Bus Station of Karad during travelling to our college from auto. It is a concept where in auto rickshaw drivers offers an safe and reliable ride to the passengers. Indeed Auto Rickshaw Services is endeavouring to solve the problems of accessibility, transparency and safety for auto rickshaw users. In other hand its focuses is also provide security, prosperity and dignity to auto rickshaw drivers.

There is no system available in Karad City for booking an auto rickshaw from doorstep of the passenger at any time. Also the drivers fare as per his own preference. Today's system is very time consuming and also there is no guarantee of routes.

To overcome all this issues there is need to design a system from which people will get affordable ride as well as get satisfaction from it. Under this service, passenger can book and locate nearby auto rickshaw $24 * 7$ through this application. This system is reliable and highly effective in local delivery.

In the present system, no work is done related to this kind of application in Karad City. This is a whole new idea for the application of the transportation system. Travelling is the basic need of humans nowadays. We are focusing on reserving an auto particularly, because there is not a single application for this purpose, whereas there are thousands of applications for cab services, bus services. To overcome the issues Auto Rickshaw services allows you to book on demand, affordable and safe rides without the hassles of getting overcharged. Simply tap and a certified driver reaches your desired pick up location within minutes

\section{LITERATURE SURVEY}

Applications can be developed on Android platform of Open Handset Alliance led by Google. Google provides simulated environment and standard development kit for developing Android applications.

The LBS application can help user to find hospitals, school, gas filling station or any other facility of interest indicated by user within certain range. Just like a GPS device its location will also be updated as soon as user changes his/her position.[1]

Android based transportation system is an application which will be used to order a transportation heavy goods anytime anywhere via vehicles.

The application of the transportation system including shifting of all the household goods at a single time. [2]

Manav Sighai, Anupam Shukla implement the location based service using Google We Services and Walk Score Transit APIS on Android phones which is useful for the multiple services that want by the user based on their location. Android Location API is used to retrieve the location information of the user.

Google Place API is service returns data about place. It has limitations that user are allowed only 1000 request in a day. 
IARJSET

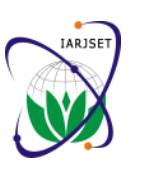

International Advanced Research Journal in Science, Engineering and Technology

National Conference on Innovative Applications and Research in Computer Science and Engineering (NCIARCSE-2017)

AGTI's Dr. Daulatrao Aher College Engineering, Vidyanagar Extension, Karad

Vol. 4, Special Issue 4, January 2017

Pubic Transportation API is used to add transit score to Then it will generate a user account and User Id as well as the application, to view public transit stoppage on map and the password will be given to the user.

to view nearby transportation route.[3]

Amol Dhumal, Amol Naikoji, Yutika Patwa, Manali Shilimkar, Prof. M.K. Nighot describe that their system allows organization to track their vehicle and get exact location. Also those companies can monitor the travelled route using Google Map API.

In that they use two units transmitting unit and monitoring unit to obtain such information regarding to their vehicle.[4]

Amit Khushwaha, Vineet Khushwaha introduce that, how the location of mobile device is obtained. Location based service can be divided into two services push services and pull services. In push services, the location of user's mobile device is retrieved. In pull service, the user decides whether and when to retrieve the location of his/her mobile service and use it in service. [5]

\section{DESIGN AND PROPOSED WORK}

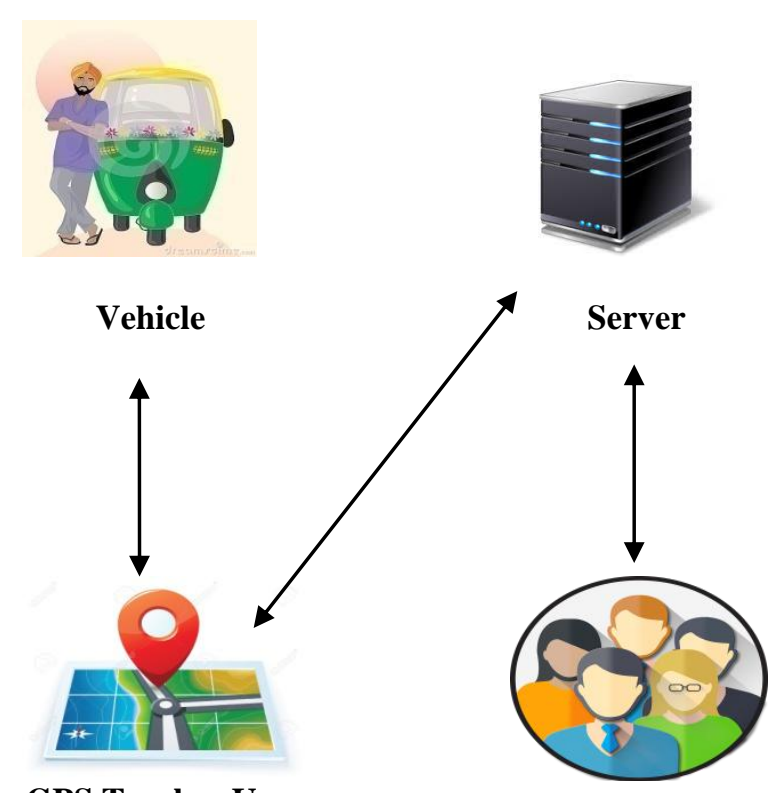

GPS Tracker User

Fig 1. System Architecture

GPS Based public transportation system uses the GPS and Android phones. As per the need of the user it tracks the nearest auto through the GPS and send confirmation as well as detailed information regarding to that auto. Also it shows the current location of the auto on Google map. The server works as mediator between the user and vehicle drivers.

This System mainly consist two parts one for the Vehicle and second one for the passenger.

For Vehicle side it consist following modules-

1. Registration: The owner of the vehicle fill his personal registration information to use this application. Then the server verify this information and if it is unique then it is stored in database.

2. Login: When user $\log$ s into the system it need to insert the User Id and Password. Server compare the information of the user to the information stored in the database. If the user identity is verified successfully then the next session will start otherwise it shows an error message on the window.

3. Perform Status: The user can perform a live as well as offline status. If he perform live status then the all the notifications regarding to the passenger request for the ride will displays on his application window. If he perform offline status no information will display.

4. Passenger Request: In this user can decide whether he wants to go for ride or not. If he is interested for ride he can start the session otherwise he can reject this request.

For Passenger it contains following module,

1. Registration: The Passenger fill his personal registration information to use this application. Then the server verify this information and if it is unique then it is stored in database.

Then it will generate a user account and User Id as well as the password will be given to the user.

2. Login: When user logs into the system it need to insert the User Id and Password. Server compare the information of the user to the information stored in the database. If the user identity is verified successfully then the next session will start otherwise it shows an error message on the window.

3. Search Vehicle: In this passenger fill the information related to ride i.e. where he wants to go and he will find the auto for the searched destination.

\section{IV.GPS TECHNOLOGY}

GPS stand for Global Positioning System is a satellite based navigation system. Now a days this is widely used in android phones. This enables GPS receivers to determine their current location, time and velocity. GPS is often used as navigation system. The result is provided in the form of a geographic position -longitude and latitude. Software application uses this to provide instruction. Today's GPS receivers are extremely accurate. Also it is feasible solution to aid travelers from getting lost. Earlier travelers used to rely on elaborate maps to track and monitor the route to their destination. It helps to track location, objects and even individuals.

\section{SHORTEST PATH ALGORITHM}

A shortest path algorithm is a program, or set of directions that can be executed to provide the shortest path between locations given certain conditions and paths. Conditions 
IARJSET

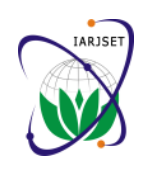

International Advanced Research Journal in Science, Engineering and Technology

National Conference on Innovative Applications and Research in Computer Science and Engineering (NCIARCSE-2017)

AGTI's Dr. Daulatrao Aher College Engineering, Vidyanagar Extension, Karad

Vol. 4, Special Issue 4, January 2017

such as traffic density, speed of travel, and others, as well as geographic obstacles can be factored in to help the algorithm execute and display the shortest path.

Shortest path algorithm is used to find the shortest distance from one node to another node. With the help of this current position is retrieved at any point. By using this current position, the distance can be determined from one node to another node.

Steps in shortest path algorithm-

\section{Step 1. Initialization}

- Assign the zero distance value to node S, label it as permanent.

- Assign to every node a distance value of infinity and label as temporary.

- Designate the node $\mathrm{S}$ as the current node.

Step2. Distance Value Update and Current Node Designation Update

Let $\mathrm{i}$ be the index of the current node.

1) Find the set $\mathbf{J}$ of nodes with temporary labels that can be reached from the current node $i$ by a link (i, j). Update the distance values of these nodes.

For each $\mathrm{j}$ belongs to $\mathrm{J}$, the distance value dj of node $\mathrm{j}$ is updated as follows

new $d j=\min \{d j, d i+c i j\}$

where cij is the cost of link (i, j), as given in the network problem.

2) Determine a node $\mathrm{j}$ that has the smallest distance value dj among all nodes $\mathrm{j}$ belongs to $\mathrm{J}$

find $\mathrm{j}^{*}$ such that $\min \mathrm{j}$ belong to $\mathrm{J} \mathrm{dj}=\mathrm{dj}{ }^{*}$

3) Change the label of node $j_{-}$to permanent and designate this node as the current node.

\section{Step 3. Termination Criterion}

If all nodes that can be reached from node $\mathrm{s}$ have been permanently labelled, then stop - we are done. If we cannot reach any temporary labelled node from the current node, then all the temporary labels become permanent we are done. Otherwise, go to Step 2.

\section{CONCLUSION}

This system offers a reliable yet affordable rides. Click for an auto and reach your destination within minutes. In case of emergency this android app will help peoples in better way. It will provide a better quality of services to the peoples and also they get satisfaction from it.

\section{ACKNOWLEDGMENT}

It is our privilege to acknowledge with deep sense of gratitude to our project guide Prof. Mahind R. N. and our Head of Department Prof. Patil A. N. whose supervision, inspiration and valuable discussion has helped us tremendously to complete our project. Their guidance proved to be the most valuable to overcome all the hurdles in the fulfilment for the project.

\section{REFERENCES}

1. Prof. S. Vanjire, Prof. Unmesh Kanchan, Prof. Ganesh Shitole, Prof. Pradnyesh Patil "Location Based Services on Smart Phone through the android application” January 2014.

2. Abhijeet A.Thakare, Gaurav G.Singhania, Krunal R.Bhongade, Sushil Yadav "Android Based Transportation System” 2016.

3. Manav Sighai, Anupam Shukla "Implementation of Location Based Services in Android Using GPS and Web Services” January 2012.

4. Amol Dhumal, Amol Naikoji, Yutika Patwa, Manali Shilimkar, Prof. M.K. Nighot "Vehicle Tracking System Using GPS and Android Operating System " April 2015.

5. Amit Khushwaha, Vineet Khushwaha "Location Based Service Using Android Mobile Operating System” March 2011. 